\title{
GALAXIES
}

\section{Slow motions in the cosmic web}

The cosmic web is the large-scale structure arising from the anisotropic collapse of the initial fluctuations of the matter density field under the effect of gravity over cosmic time. Katarina Kraljic and collaborators have produced the pictured image that shows the features of the cosmic web in the G12 field of the Galaxy And Mass Assembly (GAMA) spectroscopic survey of galaxies (Mon. Not. R. Astron. Soc., in the press; preprint available at https://arxiv.org/ abs/1710.02676).

They have used an algorithm (called DisPerSE) that exploits the topology and connectivity of the observed GAMA galaxies to generate three-dimensional density ridges using Morse theory. Filaments are shown in thick black and red lines (red being the most persistent features), while walls are shown with narrow lines in a variety of colours. Filaments are connected at nodes, which correspond to high-density peaks in the distribution of galaxies: galaxy clusters and large groups reside here. The thin, sheetlike walls surround cosmic voids.

The GAMA survey itself (S. P. Driver et al. Mon. Not. R. Astron. Soc. 413, 971-995; 2011) provides spectroscopically determined redshifts of galaxies over a large area on the sky and multi-wavelength (ultraviolet to far-infrared) coverage. The G12 field covers an area of $60 \mathrm{deg}^{2}$ across the Galactic equator, and contains nearly 100,000 galaxies over the redshift range $0.03 \leq z \leq 0.25$. In the work by Kraljic et al., galaxy groups are constructed from this sample using a friends-of-friends algorithm on the projected separations of

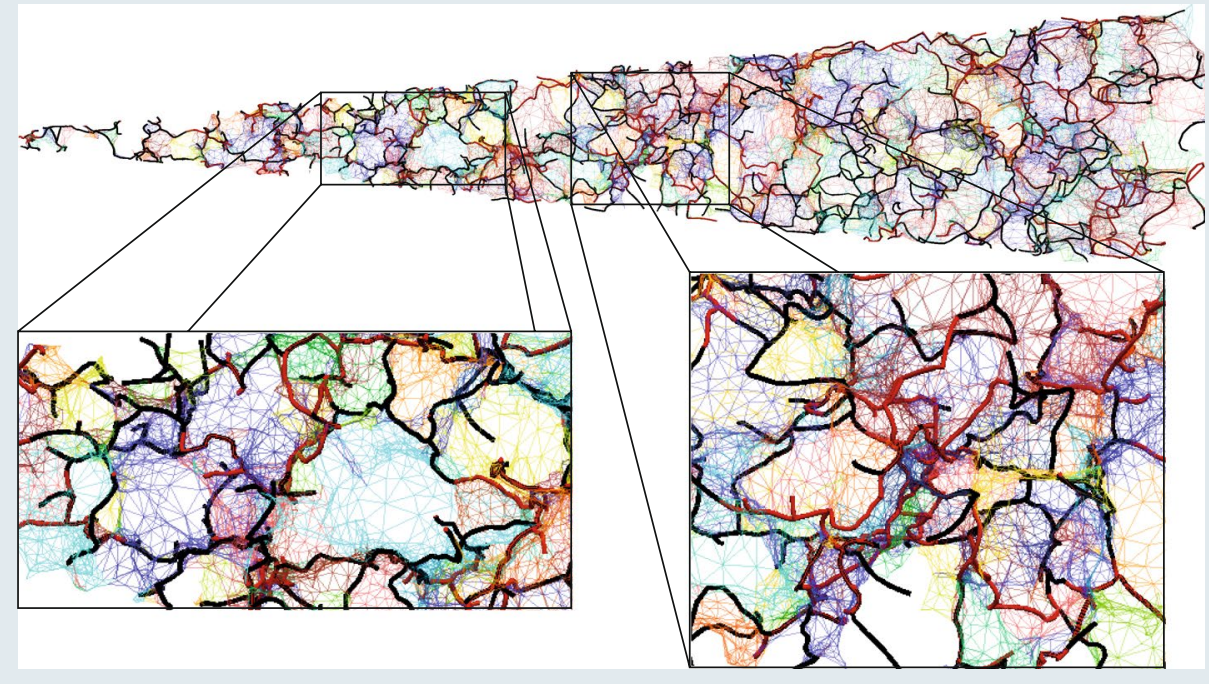

Credit: Oxford Univ. Press

the galaxies, and from this a centre of mass - and the central galaxy of each group are computed.

What, if any, is the impact of the largescale structure of the Universe on the properties of galaxies, such as their stellar mass, colour and specific star-formation rate? Kraljic et al. find that these properties do correlate with galaxies' relationships to the cosmic web. For instance, more massive galaxies are located closer to the cores of filaments and to walls, with passive galaxies (as opposed to star-forming galaxies) also following this arrangement. On the other hand, more active star-forming galaxies are farther from filaments.
The authors interpret this segregation in terms of mass and activity as a result of filamentary motions - tides - that shape galaxies. Reassuringly, state-of-the-art hydrodynamical simulations reproduce the same trends in galaxy properties with respect to the cosmic web, implying that the key astrophysics involved in our current theoretical understanding of how galaxies evolve is successfully captured.

\section{Paul Woods}

Published online: 2 November 2017 https://doi.org/10.1038/s41550-017-0315-X 